\title{
Tandem in situ monitoring for quantitative assessment of mechanochemical reactions involving structurally unknown phases
}

\author{
Stipe Lukin, Tomislav Stolar, Martina Tireli, Maria Valeria Blanco, Darko Babić, Tomislav Friščić, Krunoslav Užarević, ${ }^{*}$ \\ and Ivan Halasz*
}

We report here quantitative in situ monitoring via simultaneous PXRD and Raman spectroscopy of the mechanochemical reaction between benzoic acid and nicotinamide, affording a rich polymorphic system with four new cocrystal polymorphs, multiple phase transformations and a variety of reaction pathways. After observing polymorphs by in situ monitoring, we were able to isolate and characterize three of the four polymorphs, most of which are not accessible from solution. Relative stabilities among the isolated polymorphs at ambient conditions were established by slurry experiments. Using two complementary methods for in situ monitoring enabled quantitative assessment and kinetic analysis of each studied mechanochemical reaction, even when involving unknown crystal structures, and short-lived intermediates. In situ Raman monitoring was introduced here also as a standalone laboratory technique for quantitative assessment of mechanochemical reactions and understanding of mechanochemical reactivity. Our results provide an important step toward a complete and high-throughput quantitative approach to mechanochemical reaction kinetics and mechanisms, necessary for the development of the mechanistic framework of milling reactions.

\section{Introduction}

Mechanochemical milling is emerging as a versatile synthesis techniques for a variety of chemical transformations, ${ }^{1,2}$ including synthesis and screening of cocrystal forms ${ }^{3-7}$ and polymorphs. ${ }^{8-14}$ Despite the long history of mechanochemistry ${ }^{15,16}$ and the use of milling in various branches of chemical and materials processing, ${ }^{17-20}$ the underlying mechanistic details of these reactions are only now becoming accessible, enabled by the recent development of in situ reaction monitoring techniques based on powder X-ray diffraction (PXRD), ${ }^{21}$ Raman spectroscopy ${ }^{22}$ and a combination of the two. ${ }^{23}$ These advanced techniques have revealed a surprising level of complexity in milling reac-

[*] S. Lukin, T. Stolar, M. Tireli, Dr. D. Babić, Prof. T. Friščić, Dr. K. Užarević, Dr. I. Halasz

Ruđer Bošković Institute, Bijenička c. 54, 10000 Zagreb (Croatia)

E-mail: ivan.halasz@irb.hr

krunoslav.uzarevic@irb.hr

Dr. M. V. Blanco,

ESRF - the European Synchrotron, Grenoble (France)

Prof. T. Friščić,

Department of Chemistry, McGill University, Montreal (Canada)

Supporting information for this article is available on the WWW under http://dx.doi.org/10.XXXX/XXXX

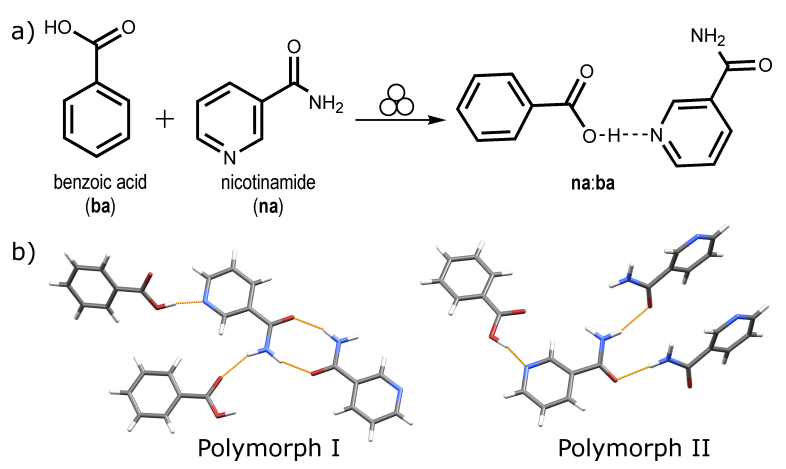

Fig. 1 a) Mechanochemical cocrystallisation of benzoic acid and nicotinamide, and b) hydrogen-bonding in crystal structures of polymorphs I and II.

tions, involving crystalline and amorphous intermediates, as well as multi-step reaction mechanisms that can be sensitive to slight variations in reaction conditions. ${ }^{24}$

While qualitative evaluation of mechanochemical reaction is now generally available, a persistent major challenge in mechanochemical reactions still remains the absence of their mechanistic understanding, primarily resulting from the inability of quantitative evaluation of mechanochemical reaction kinetics. Quantitative in situ reaction monitoring has thus far been limited to synchrotron PXRD, ${ }^{25,26}$ and only to those systems where crystal structures of all participating phases were known, enabling Rietveld analysis. Consequently, a reaction product or an intermediate with an unknown crystal structure represents an insurmountable problem for quantitative Rietveld analysis as well as addressing the chemical composition of any amorphous phases that can easily be generated during milling. ${ }^{25,26}$

To remedy the currently very scarce mechanistic understanding of mechanochemical milling reactions, accumulation of knowledge of reaction profiles for a large number of reactions and reaction types will be required. While Raman spectroscopy has been used to monitor mechanochemical reactions in situ and ex situ, current approaches mostly relied on extracting the reaction profile from changes in the intensity of a single peak. ${ }^{27,28}$ Clearly, continuing in this fashion would present a tedious task for collecting sufficient kinetic data for a variety of mechanochemical reactions conducted under a variety of reaction conditions. Additionally, ex situ approach is not suitable for self-propagating and air-sensitive reactions, as well as the use of volatile additives. ${ }^{22}$

Herein, we describe tandem in situ mechanochemical reaction monitoring, with time-resolution in seconds, combined with a 
high-throughput analysis to extract reaction concentration profiles. Additionally, we were able to use Raman in situ monitoring to quantitatively assess reaction profiles even in situations where Rietveld refinement was not possible since not all of the participating phases have been structurally characterized which hindered . Our approach is verified on a challenging model system that involves cocrystallization of pharmaceutically relevant benzoic acid (ba) with the model active pharmaceutical ingredient nicotinamide (na) in a dynamic reaction environment involving multiple polymorphic transformations, fast kinetics, novel crystalline phases and short-lived intermediates (Scheme 1).

\section{Experimental Section}

Mechanochemical reactions were carried out by using IST500 (InSolido Technologies, Croatia) or MM301 (Retsch, Germany) mixer mills operating at $30 \mathrm{~Hz}$ in translucent and amorphous reaction vessels made from polymethylmetacrylate (PMMA). Two halves of the vessel snapped upon closure to form a leak proof seal. Liquids for liquid-assisted grinding were added using Gilson automated micro pipette.

Tandem in situ monitoring experiments were conducted in the experimental hutch of the ESRF air-conditioned to $20^{\circ} \mathrm{C}$. The X-ray beam $(\lambda=0.195 \AA)$ and the Raman laser $(\lambda=785 \mathrm{~nm})$ focus were positioned to approximately coincide during milling in order to collect data on the same portion of the reaction mixture. X-ray radiation wavelength of $0.195 \AA$ was selected using a multilayer monochromator. Diffraction data were recorded on a Dectris Pilatus CdTe 2M detector positioned $1067 \mathrm{~mm}$ from the sample. Radial integration of the raw diffraction images was performed using PyFAI. ${ }^{29}$ Exposure time for each pattern was $5 \mathrm{~s}$ while time resolution between consecutive patterns was ca. $6.5 \mathrm{~s}$. Time resolution of Raman spectra was typically $10 \mathrm{~s}$.

In situ monitoring was conducted at the new ID31 high-energy beamline of the European Synchrotron Radiation Facility (ESRF) using a ball mill custom modified to allow for simultaneous Xray diffraction ${ }^{30}$ and Raman monitoring. ${ }^{22}$ Reactants, $1 \mathrm{mmol}$ $(122 \mathrm{mg}$ ) of na and $1 \mathrm{mmol}(122 \mathrm{mg})$ of ba were weighed in separate halves of the reaction vessel to avoid contact before milling. As milling media two $7 \mathrm{~mm}$ stainless steel balls were used, each weighing $1.4 \mathrm{~g}$. Each experiment was started with a new, pristine reaction vessel to reduce the risk of possible contamination with crystal seeds from earlier experiments. ${ }^{31}$ Tandem in situ monitoring was performed on neat grinding as well as on LAG reactions involving as additives water or simple aliphatic alcohols (methanol, ethanol, 1-propanol, 1-butanol) added in the amount of $0.1 \mathrm{mmol}$.

Where possible, quantitative Rietveld refinement ${ }^{32}$ was performed on in situ collected powder diffraction patterns. Crystal structures of III and IV are not known, which limited the use of Rietveld refinement of data sets where they had formed. The first diffraction pattern was sometimes excluded from the analysis due to it still having a non-uniform mixture composition. The Rietveld refinement on a series of patterns was performed in an automated fashion using the command-line version of Topas, usually always starting from the same input file which was prepared so that each phase had its scale factor between its lowest and highest observed values for that milling experiment. In our case, this proved a better approach then sequential refinement, which takes the output of the previous refinement as an input for the next one, which often resulted in meaningless parameter values toward the end of the run. Rietveld refinements included refinement of the parameters for the shifted Chebyshev polynomial used to describe the background parameters, parameters contributing the peak position and shape (contribution to the lorenzian and gaussian full widths at half maximum, zero shift, unit cell parameters). The structure models were taken as solved from powder diffraction data (see section S6). No instrument contribution to the peak shape was assumed. In each cycle of Rietveld refinement, after reaching convergence, weight fractions of refined phase were output to a separate file. All calculations were performed using the program Topas.

Figures showing two-dimensional time-resolved PXRD or Raman data were created using the program Mathematica with the help of the SciDraw package. ${ }^{33}$ Background of each diffraction pattern was subtracted prior to plotting using the SonneveldVisser ${ }^{34}$ algorithm which we have implemented in Mathematica.

Laboratory in situ Raman monitoring was performed using an IST500 mixer ball mill from InSolido Technologies (Croatia) operating at $30 \mathrm{~Hz}$. Time-resolved in situ Raman spectra were collected as described previously. ${ }^{22}$ Raman monitoring was performed using a portable Raman system with a PD-LD (now Necsel) BlueBox laser source (excitation wavelength $785 \mathrm{~nm}$ ) equipped with B\&W-Tek fiber optic Raman BAC102 probe, and coupled with OceanOptics Maya2000Pro spectrometer. Probe was positioned under the milling vessel using a movable stand, so to place a focus of the laser $1 \mathrm{~mm}$ inside of the vessel. In each experiment, after the monitoring was finished, the sample was taken out and analyzed by PXRD.

Reaction vessel subtraction. Raman spectrum of the PMMA reaction vessel was taken in the same experimental conditions in which all mechanochemical experiments were performed (mill operating at $30 \mathrm{~Hz}$ vessel being mounted at the same distance from the probe). For subtracting the vessel contribution from experimental spectra, the peak at $2955 \mathrm{~cm}^{-1}$, corresponding to the $\mathrm{C}-\mathrm{H}$ bond stretching of PMMA, was used as the reference for scaling. From the ex situ analysis it was found that it does not overlap with any peak of reactant and product phases. Spectral range of $2889-3007 \mathrm{~cm}^{-1}$ (61 points) was background corrected with Sonneveld-Visser ${ }^{34}$ algorithm in both the experimental and the vessel Raman spectra. The intensities of the experimental spectrum in the range $2933-2968 \mathrm{~cm}^{-1}$ (19 points) were divided by those of the vessel spectrum. The mean quotient value was used as the scaling factor by which the whole vessel spectrum was multiplied and subtracted from the experimental one. This procedure was repeated for all experimental spectra to provide pure spectra without the vessel contribution.

Analysis of Raman spectra. For acquisition of the Raman spectra of pure na and ba, $2 \mathrm{mmol}$ of each was grinded separately in the same experimental conditions. Average spectrum 
was taken as the pure spectrum for each of these two phases. The spectra of pure I, II and III were taken from the in situ experiments, from the parts of the reaction where PXRD showed that they were present as a pure phase. All spectra were baseline corrected using asymmetric least squares smoothing ${ }^{35}$ (AsLS) method.

Phase IV could be observed as fleeting intermediate in LAG reactions with alcohols. Since it could not be isolated as a pure phase, its Raman spectrum could not be obtained as for I, II and III. Multivariate curve resolution - alternating least squares ${ }^{36}$ (MCR-ALS) was performed on the data from LAG reactions with ethanol, 1 propanol and 1 butanol in an attempt to estimate the Raman spectrum of IV. Non-negativity was enforced to both mole fraction and spectral profiles. Additionally, sum of the mole fractions was required to be 1 . Prior to the analysis all spectra were normalized. The obtained spectrum of IV was very similar to III, which also agreed well with the findings from PXRD analysis. However, when other experimental spectra were analyzed with this spectrum included, there were obvious artifacts in the mole fraction profiles. Actually, it was found that by using the spectrum of III instead of IV, more sensible results are obtained, although it looks clear that the transient intermediate IV can not be III.

Reaction evolution profiles were analyzed by using the restrained classical least squares method implemented in MCR-ALS $\mathrm{GUI}^{37}$, including the closure and non-negativity constraints together with an equality constraint for the spectra of pure phases. Prior to the analysis all experimental spectra and the spectra of pure phases were normalized. Standard deviations for mole fractions estimated from the $k$-th experimental spectrum were computed from the covariance matrix:

$$
\operatorname{Cov}=\sigma^{2}\left(\mathbf{S S}^{\mathrm{T}}\right)^{-1}
$$

where $\mathbf{S}$ is the matrix with row-wise spectra of pure components and $\sigma^{2}$ is the variance of the residuals for the $k$-th experimental spectrum

$$
\sigma^{2}=\frac{\mathbf{e}^{\mathbf{T}} \mathbf{e}}{n-p}
$$

and $\mathbf{e}$ is the vector of residuals, $n$ is the number of spectrum points and $p$ is the number of estimated regression coefficients (mole fractions).

This approach gave very good quantitative results for evolution of reaction mixture that agreed well with the quantitative Rietveld analysis of PXRD data. Example quantitative Raman analysis is given in Section S4 in Figs. S9-S20.

Laboratory powder X-ray diffraction (PXRD) patterns were collected using a Philips PW 3710 diffractometer with a $\mathrm{CuK} \alpha$ radiation, tension $40 \mathrm{kV}$, and current $40 \mathrm{~mA}$, with a flat plate sample on a zero background in Bragg-Brentano geometry. The patterns were collected in the $2 \theta$ range of $4^{\circ}$ to $40^{\circ}$ with a step size of $0.02^{\circ}$ and $1.0 \mathrm{~s}$ counting per step.

Thermal analysis. Differential scanning calorimetry (DSC) experiments were performed on Discovery DSC (TA Instruments). Around $2 \mathrm{mg}$ of each sample was put in aluminum crucible. All samples were measured in the range of $25^{\circ} \mathrm{C}$ to $400{ }^{\circ} \mathrm{C}$ with a heating rate of $5.0^{\circ} \mathrm{C} / \mathrm{min}$. Experiments were performed in an inert $\mathrm{N}_{2}$ atmosphere. Thermal stability of na:ba cocrystal polymorphs were determined from TGA experiments (Discovery TGA, TA Instruments). Around $5 \mathrm{mg}$ to $10 \mathrm{mg}$ of each sample was put in platinum HT pan type. All samples were heated at the rate of $5.0^{\circ} \mathrm{C} / \mathrm{min}$ up to $400{ }^{\circ} \mathrm{C}$. Experiments were performed in an inert $\mathrm{N}_{2}$ atmosphere.

Fourier-transform infrared attenuated total reflectance (FTIR-ATR) measurements were performed on a Perkin-Elmer Spectrum Two instrument equipped with a diamond crystal Quest ATR Accessory. The measurements were taken in the range of $4000-400 \mathrm{~cm}^{-1}$.

\section{Results and discussion}

Although na and ba are ubiquitous molecules which have complementary hydrogen-bonding functionalities, and have been widely investigated as components of cocrystals, ${ }^{38-47}$ their mutual cocrystallization has not yet been described. The herein conducted mechanochemical reactions of the 1:1 stoichiometric mixture of solid na and ba, either by liquid-assisted grinding (LAG) $^{12,48}$ or by neat grinding (NG), led us to discover four new crystalline phases in this cocrystal system. The phases I and II were structurally characterized as polymorphs of the 1:1 na:ba cocrystal while crystal structures of phases III and IV currently remain unknown. The crystal structures of both I and II were solved from PXRD data collected on the mechanochemically prepared samples (Section S6 in the Supporting Information). In both polymorphs the $-\mathrm{COOH}$ group of ba forms a hydrogen bond towards the pyridine nitrogen of na. Whereas in I two molecules of na associate through hydrogen bonds to form dimers, in II the na molecules are assembled into an extended hydrogen-bonded network (Scheme 1b).

Our attempts to solve the crystal structure of III from PXRD data have been unsuccessful, as were attempts to grow single crystals of III from solution (Section S7). PXRD analysis however, shows that III is obtained as a pure phase starting from the 1:1 na:ba mixture. Spectroscopic (Figs. S37, S38) and thermal analyses (Fig. S41) are consistent with III being a 1:1 cocrystal of na and ba and, therefore, a polymorph of I and II. In contrast, the phase IV was observed only during in situ monitoring experiments and was never obtained as a pure phase.

Tandem in situ monitoring was conducted at the new ID31 high-energy beamline of the European Synchrotron Radiation Facility (ESRF) using a mixer ball mill custom modified to allow for tandem X-ray diffraction ${ }^{30}$ and Raman monitoring. ${ }^{22}$ Reactions were conducted in amorphous and transparent polymethylmetacrylate reaction vessels oscillated at $30 \mathrm{~Hz}$ in a horizontal plane. The X-ray beam was passed through the bottom of the reaction vessel while the incident laser for Raman spectroscopy was set to enter the vessel from below and to coincide with the X-ray beam. Reactants, $1 \mathrm{mmol}(122 \mathrm{mg}$ ) of na and $1 \mathrm{mmol}$ (also $122 \mathrm{mg}$ ) of ba, were weighed in separate halves of the reaction vessel to avoid contact before milling. As milling media two $7 \mathrm{~mm}$ stain- 

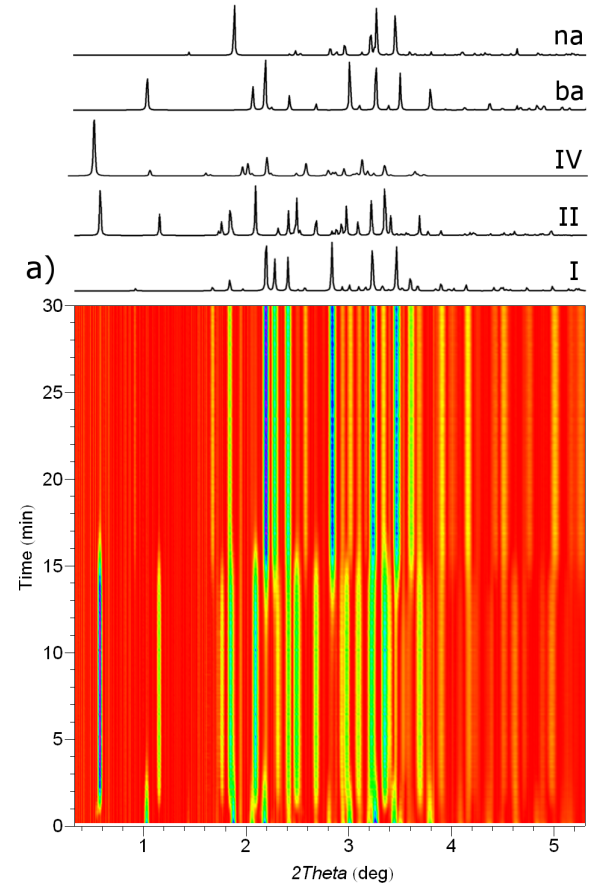
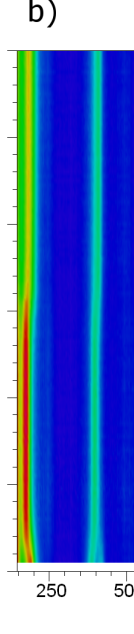

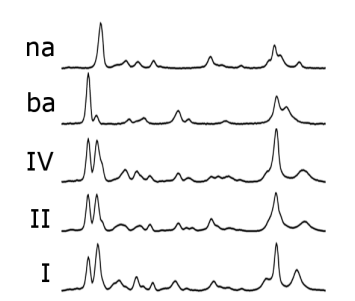

c)
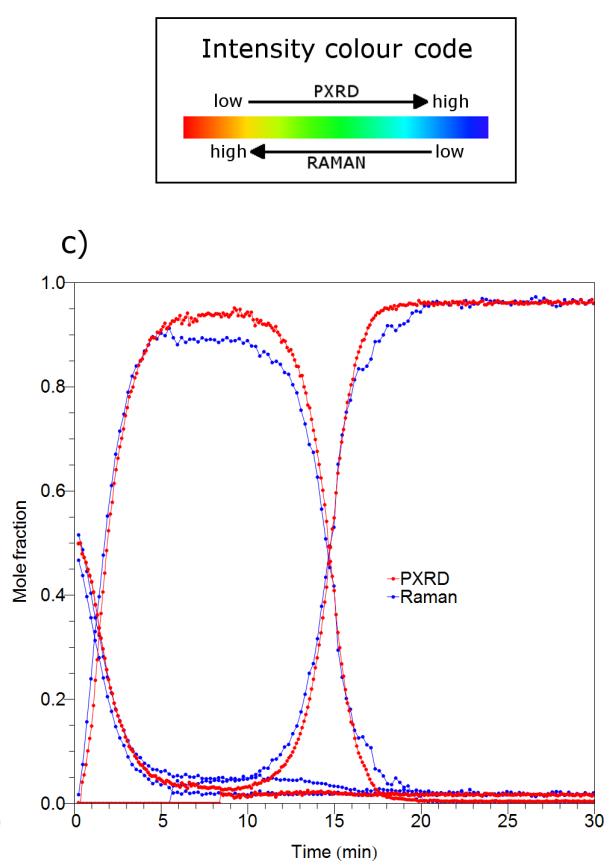

Fig. 2 Tandem in situ monitoring by (a) PXRD and (b) Raman spectroscopy of the LAG reaction of the 1:1 na:ba mixture using water. PXRD patterns and Raman spectra of pure components are given on top. The shown portion of Raman spectra was used in quantitative analysis. (c) Comparison of the evolution of the reaction mixture composition derived from time-resolved PXRD patterns and Raman spectra.

less steel balls were used, each weighing $1.4 \mathrm{~g}$. Each experiment was started with a new, pristine reaction vessel to reduce the risk of possible contamination with crystal seeds from earlier experiments. ${ }^{31}$ LAG reactions included $0.1 \mathrm{mmol}(10 \mathrm{~mol} \%$ relative to the na:ba mixture) of water or simple aliphatic alcohols as liquid additives.

\subsection{Qualitative analysis}

A LAG reaction using water $(2.0 \mu \mathrm{L})$ as the liquid additive yielded a stepwise reaction with polymorph I as the final product which formed from polymorph II ca. 15 min into milling (Figs. 2, S6). The formation of II was here preceded by the appearance of a tiny amount of IV. Replacing water with methanol (MeOH, $4.1 \mu \mathrm{L})$ led to an immediate formation of the short-lived phase IV, followed by the formation of II (Figs. 3a, S7). The reaction was complete within ca. $6 \mathrm{~min}$, and polymorph II was stable upon further grinding (Fig. S2). In repeated experiments, polymorph II was stable even up to $3 \mathrm{~h}$ milling. The LAG reaction using ethanol (EtOH, $6.2 \mu \mathrm{L}$ ) also began with immediate formation of IV, which persisted for up to ca. $2 \mathrm{~min}$ of milling (Fig. S3). It was followed by phase II, which transformed into I after ca. 10 min milling. LAG reactions involving 1-propanol (1-PrOH, $7.5 \mu \mathrm{L}$ ) (Fig. S4) or 1-butanol (1-BuOH, $9.2 \mu \mathrm{L}$ ) both led to III as the final product. These two reactions also proceeded via rapid formation of phase IV, followed by phase II which, ultimately, over a period of ca. 10-20 minutes, transformed to phase III (Fig. 4).

Finally, we have performed tandem in situ monitoring under neat grinding conditions. Similar to LAG with water and EtOH, NG after 60 min milling also yielded $I$ as the final pure product (Figs. 5, S8). However, in situ monitoring revealed a significantly slower, stepwise reaction where the phase III was formed initially, and transformed into phase I after ca. 20 min milling.

\subsection{Polymorph stability}

Slurry experiments revealed that $\mathbf{I}$ is the thermodynamically stable form of the na:ba cocrystal at ambient conditions, followed by phases III and II in decreasing order of stability. Based on its fast formation and fleeting existence, we surmise that IV is the least stable among the four herein reported phases. The observed mechanochemical reaction pathways exhibit stepwise reaction mechanism that lead to increasingly stable phases in consistence with the Ostwald's rule of stages. ${ }^{49}$ Once isolated, phases I, II and III were stable for months.

\subsection{Quantitative assessment and kinetic analysis}

While tandem PXRD and Raman in situ monitoring experiments are readily compared by plotting on the same time scale, quantitative assessment not only provides detailed insight to species involved in the reaction but also permits its kinetic modeling. Quantitative assessment by PXRD monitoring was possible only in systems where crystal structures of all participating phases were known enabling Rietveld refinement, in a similar manner as described previously. ${ }^{6,21,26,50}$ Example Rietveld refinement plots are given in Supporting Information section S3. On the other hand, quantitative assessment via Raman monitoring is more versatile, particularly if participating phases can be isolated as pure phases.

In kinetic assessment, one caveat should be kept in mind. Namely, the reaction mixture is warming up during milling ${ }^{28,51}$ which could influence reaction kinetics as the reaction is oc- 


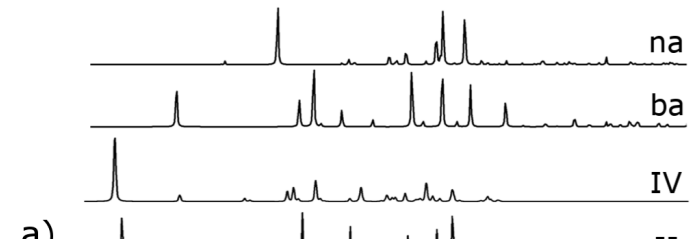

a)
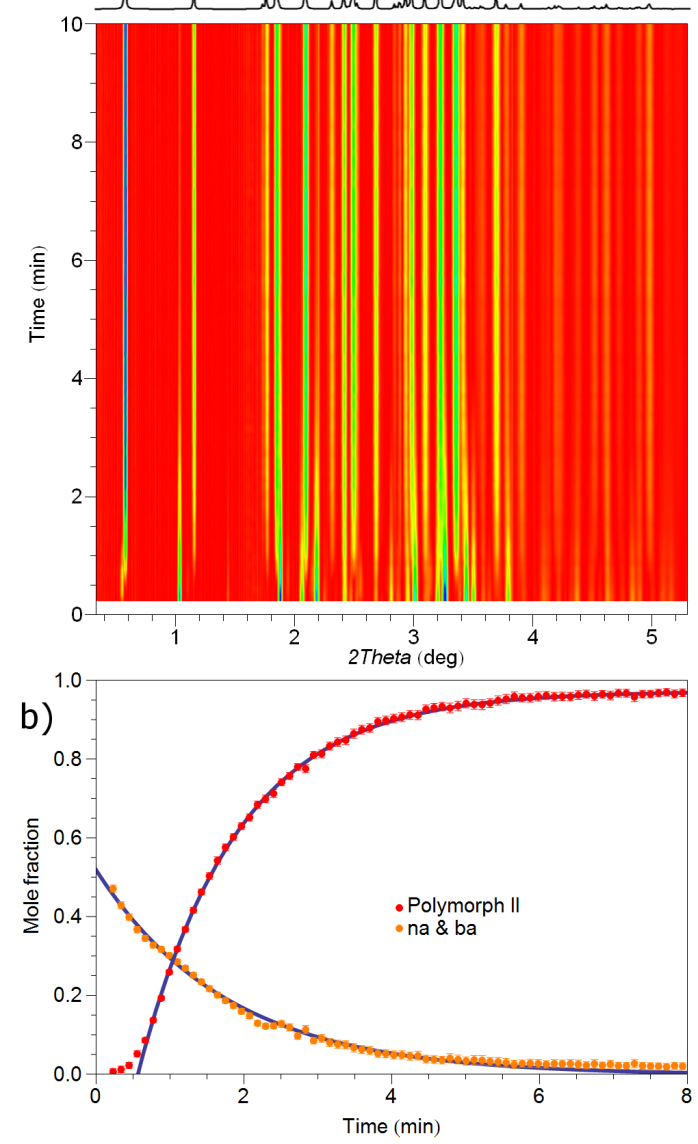

Fig. 3 (a) First 10 min of in situ PXRD monitoring of a LAG reaction using $0.1 \mathrm{mmol}(4.1 \mu \mathrm{L})$ of methanol. Intermediate IV can be observed in the first minute of milling. Calculated diffraction patterns are displayed on top. (b) Reaction profile derived from Rietveld refinement. Both curves fit the first-order reaction rate.

curring. Recently, we have shown that even mild temperature changes, achieved by preheating the milling assembly, can a have a dramatic influence on reaction kinetics and mechanism. ${ }^{26}$ Here, all the reactions were conducted under the same reaction conditions which allows us the possibility to compare different reactions, even though the reaction kinetics in each individual reaction may have been changing during milling.

The Raman spectra were analyzed by using the spectra of reactant components and of the recognized polymorphic species I, II and III, all recorded under the actual experimental conditions. Most of the spectra were successfully and completely resolved by using invariable spectral components and only reactions involving IV remained partially resolved. The concentration profiles obtained from time-resolved Raman spectra are in very good agreement with those obtained by Rietveld analysis of PXRD data (Fig. 2c). Taking into account that Raman spectra of polymorphs I,
II and III are sufficiently different, this indicates that under the present experimental conditions, cocrystal formation and polymorphic transformations do not involve a detectable amount of an amorphous phase. High crystallinity is also reflected in stable diffraction peak shape as milling progresses. In general however, a discrepancy in quantitative assessments via PXRD and Raman monitoring could be expected since PXRD is sensitive to bulk crystalline phases while Raman spectroscopy is collecting the scattering signal from all of the sample. In such cases, Raman monitoring could be more informative of mechanochemical reaction mechanism then PXRD since many reactions have a significant amorphous component. ${ }^{25,26}$

For phases that cannot be obtained pure and stable, as was the case here for phase IV, we used multivariate curve resolution alternating least squares ${ }^{36}$ (MCR-ALS) to estimate the spectrum of IV. However, the estimated spectrum of phase IV was strikingly similar to the spectrum of III leading to strong correlations between the two in a restrained classic least-squares approach to derive reaction profiles. To avoid correlations between III and IV, we have used the spectrum of III to model also the reaction profile of IV. The similarity between spectra of III and IV, as well as the similarity between PXRD patterns of III and IV, indicates that these two phases have very similar crystal structures.

Quantitative reaction profiles allowed us to apply solid-state kinetic models ${ }^{52}$ in order to describe the mechanistic background of these reactions. Application of these models to mechanochemical milling reaction is not straightforward since these kinetic models were derived primarily for static solid reaction mixtures in contrast to reaction mixtures where particles are undergoing constant breaking and recrystallization as well as stirring by the milling media. ${ }^{27}$ At this point, it is safer to avoid kinetic models that stipulate strong assumptions, as for example the popular KJMA kinetic model ${ }^{53,54}$ (also known as the Avrami, AvramiErofe'ev, JMAEK or Johnson-Mehl model), but rather to use simpler order-based kinetic models ${ }^{52}$ or the Prout-Tompkins equation. ${ }^{55,56}$

Focusing first on the depletion of reactants na and ba, we find it in all LAG reaction (Fig. 6) as well as in NG to be best described by a first-order reaction rate law given by the equation:

$$
x=x_{\infty}+\left(x_{0}-x_{\infty}\right) \mathrm{e}^{-k\left(t-t_{0}\right)}
$$

where $x, x_{\infty}$ and $x_{0}$ represent average mole fractions of na and ba at times $t, \infty$ and $t_{0}$, respectively. Constant $k$ is the reaction rate constant and $t_{0}$ accounts for the possible time delay from the initiation of milling. Ideally, $x_{0}=0.5, x_{\infty}=0$, and $t_{0}=0$ but we have allowed a discrepancy from these values to account for the time required for the reaction mixture to become homogeneous.

The LAG reaction using water allows us to compare kinetic modeling on both PXRD and Raman monitoring (Fig. 7). The depletion profiles derived from both techniques led to almost identical first-order reaction rate constants (Table 1) confirming the correspondence between quantitative assessments derived from in situ Raman and PXRD monitoring. Rates of reactant consumption are considerably faster in LAG reactions as compared to NG (Table 1, Fig. S5), consistent with the well-known accelerating ef- 

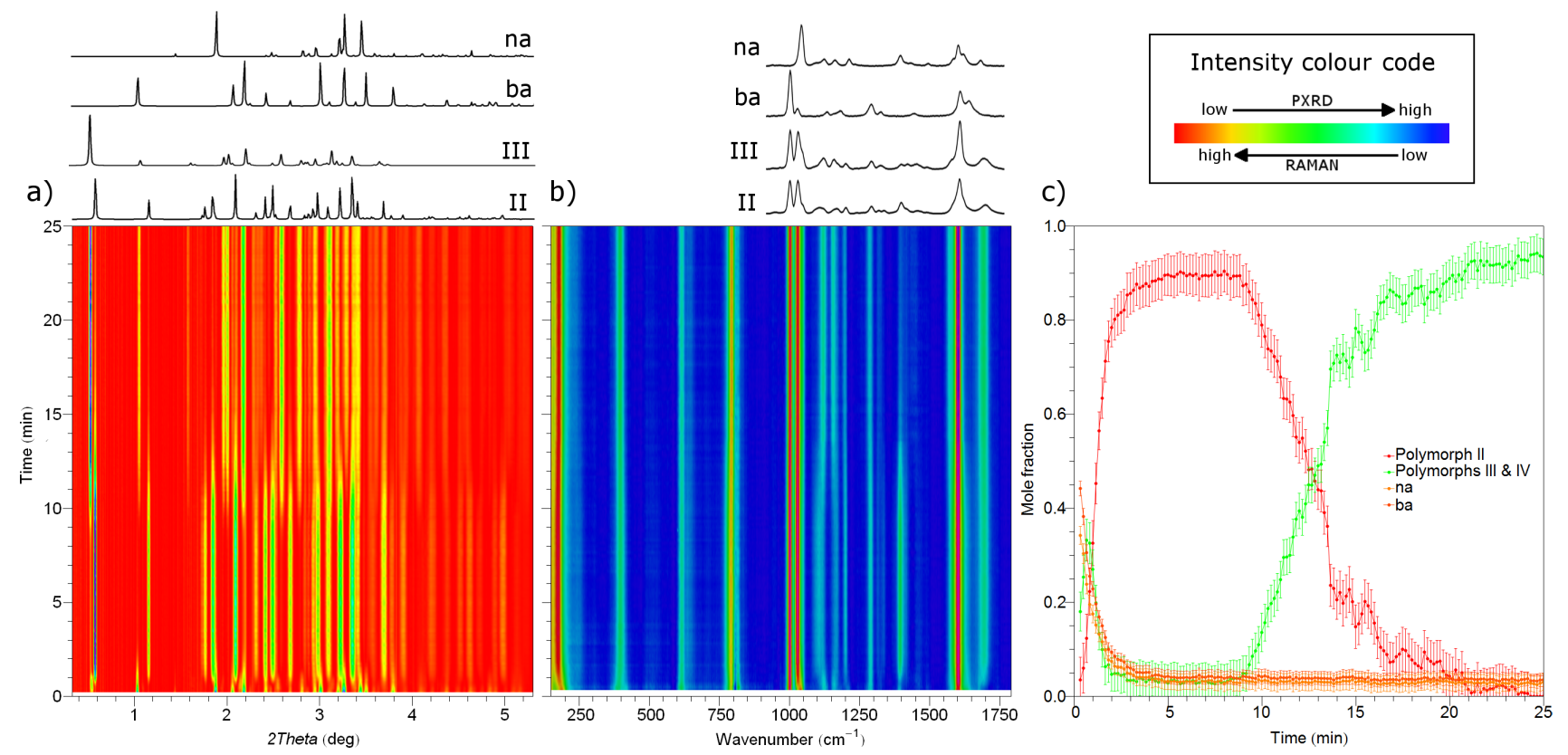

Fig. 4 Tandem in situ monitoring by (a) PXRD and (b) Raman spectroscopy of a LAG of 1:1 na:ba using 10 mol\% of 1-butanol. Diffraction patterns and Raman spectra of pure phases are given on top of time-resolved figures. The shown portion of Raman spectra was used in quantitative analysis. c) Evolution of the reaction mixture composition derived from time-resolved Raman spectra in b). Error bars correspond to twice the standard deviations as obtained from the refinement procedure.

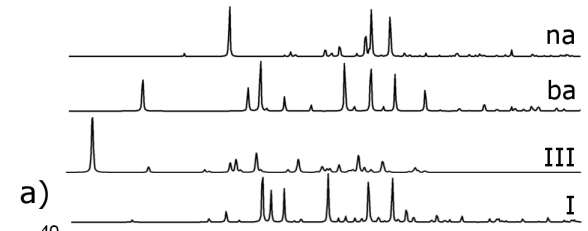

b)

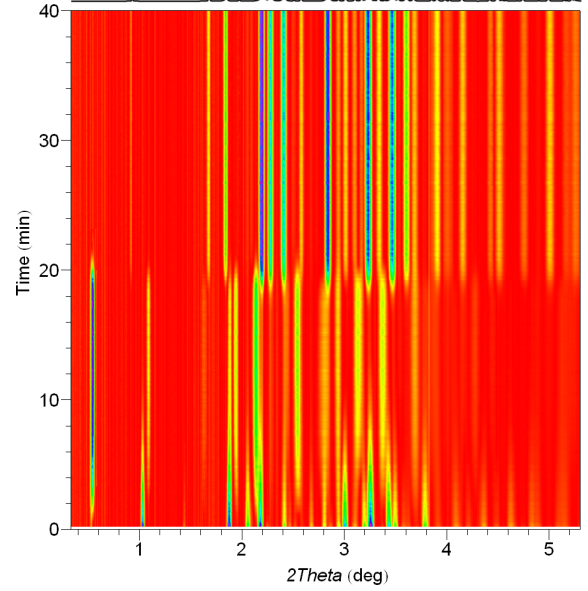

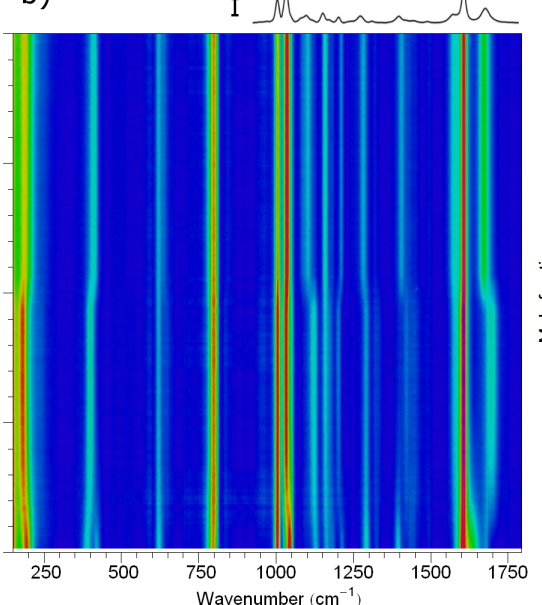

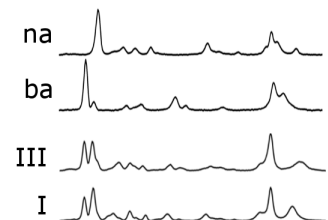

c)
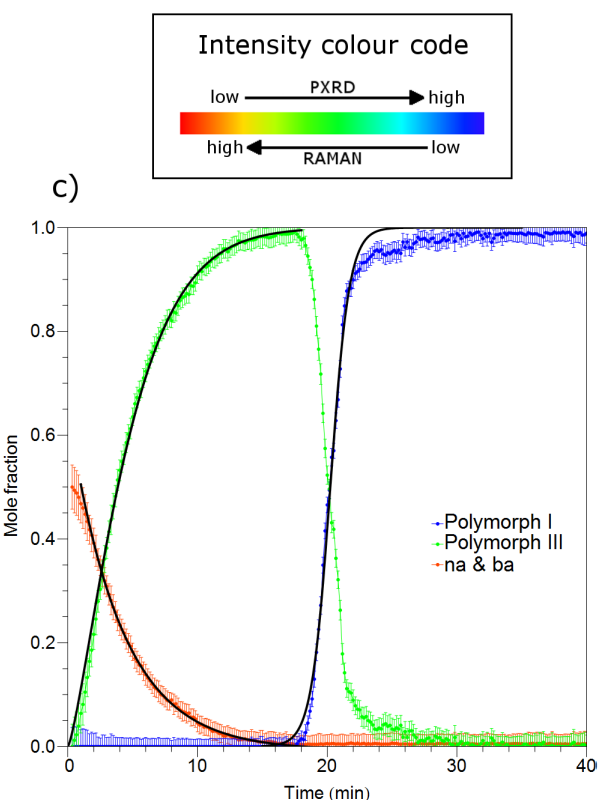

Fig. 5 Tandem in situ monitoring by (a) PXRD and (b) Raman spectroscopy of NG of 1:1 na:ba mixture. Diffraction patterns and Raman spectra of pure phases are given on top of time-resolved figures. The shown portion of Raman spectra was used in quantitative analysis. c) Evolution of the reaction mixture composition derived from time-resolved Raman spectra in b). The fitted curves are displayed in black. Reactant depletion and formation of III are described with a first-order reaction rate and the final III $\longrightarrow$ I transformation with the PT model. Error bars correspond to twice the standard deviations as obtained from the refinement procedure.

fect of liquid additives on mechanochemical reactivity. ${ }^{6,21,48}$ The alcohols (added in the amount of $10 \mathrm{~mol} \%$ ) show a constant increase in reaction rate constants while LAG with water seems to be slightly faster then LAG with $\mathrm{MeOH}$.

Formation of II in the LAG reaction with water can be well fitted with the first-order reaction rate, the same as for reactant de- pletion. The subsequent II $\longrightarrow$ I transformation exhibits a symmetrical and sharp sigmoidal curve that is well described by the Prout-Tompkins (PT) model ${ }^{55}$ which assumes a reaction rate proportional to both the amount of the remaining reactant (in this case the mole fraction of phase II, $x_{\text {II }}$ ) and the amount of the already formed product (mole fraction of phase I, $x_{\mathbf{I}}$ ). Assuming 
Table 1 Reaction rate constants for the first-order depletion of na and ba derived from in situ Raman monitoring (unless stated otherwise).

\begin{tabular}{l|c|c|c|c|c|c|c}
\hline & \multicolumn{3}{|c|}{ | Liquid (10 mol\%) } \\
\hline & NG & water & IeOH & EtOH & 1-PrOH | 1-BuOH \\
\hline$k / \mathrm{min}^{-1}$ & $0.249(2)$ & $0.69(2), 0.68(1)^{a}$ & $0.60(1)$ & $0.68(2)$ & $0.82(2)$ & $1.17(1)$ \\
\hline
\end{tabular}

${ }^{a}$ From in situ PXRD monitoring.

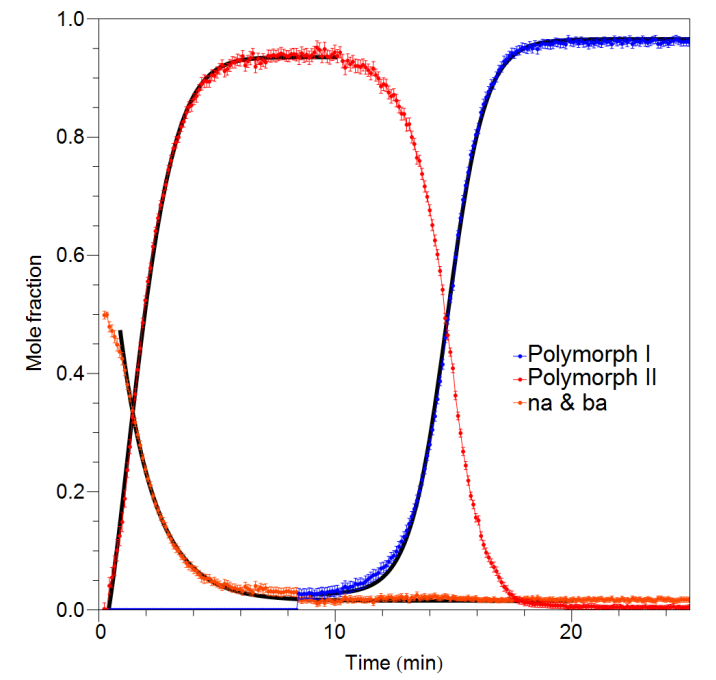

Fig. 6 Fitting the concentration profiles obtained from time-resolved PXRD monitoring for the LAG reaction using water. Depletion of reactants and formation of II is described by the first-order reaction rate while the formation of $I$ is described by the Prout-Tompkins model. Fitted curves are shown with black lines. Error bars correspond to one standard deviation as obtained from the Rietveld refinement procedure.

$x_{\mathbf{I}}=1-x_{\text {II }}$ we get:

$$
\frac{\mathrm{d} x_{\mathbf{I}}}{\mathrm{d} t}=k x_{\mathbf{I}} x_{\mathbf{I I}}=k x_{\mathbf{I}}\left(1-x_{\mathbf{I}}\right) .
$$

For the NG reaction, formation of III follows the same firstorder reaction rate as the depletion of reactants. The transformation of III to I commences not before all of na and ba have completely reacted (Fig. 5c) and exhibits a symmetrical sigmoidal curve that fits best the Prout-Tompkins kinetic model. For NG however, the PT model could not describe that well the initially sharp formation of I and slowing down of the reaction toward the end.

The formation of I from either II or III is an autocatalytic nucleation-and-growth process, as indicated by symmetrical sigmoidal growth curves describing the formation of I. On the other hand, the rate of II $\longrightarrow$ III transformation seems to be constant corresponding to a zeroth-order reaction rate law (Figs. 4c, S4). This transformation was notably slower then others and the coexistence of the two phases could be observed even for $20 \mathrm{~min}$ (Fig. 4).

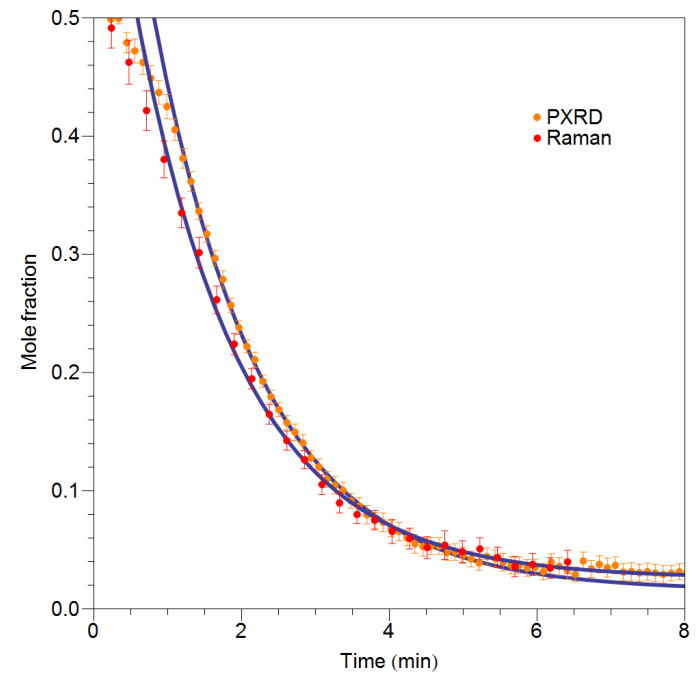

Fig. 7 Comparison of the first-order kinetic modeling of reaction profiles derived from PXRD and Raman monitoring for the LAG reaction using water. The corresponding reaction-rate constants are given in Table 1.

\subsection{The effect of liquid additive on polymorph selectivity}

The formation of phase IV seems to be kinetically favored in the presence of alcohols and water as milling additives in all LAG reactions. It is formed immediately upon milling but is soon followed by the formation of polymorph II. Stability of polymorph II is dependent on LAG conditions employed, where it can remain stable upon further milling or it can convert to either I or III. In LAG reactions, higher alcohols stabilised III while methanol stabilised II. However, the increase in molecular weight on going from methanol to 1-butanol increased the ratio of the added liquid volume to the mass of solid reactants, $i$. $e$. the $\eta$-value. ${ }^{57}$ Inspired by the recent report from the Jones group, where considerable control over cocrystal polymorphism was achieved simply by changing $\eta,{ }^{13}$ we decided to investigate whether the herein observed reactivity was due to specific interactions between the liquid additive and crystalline particles ${ }^{11,58}$ or was it related to the volume of the added liquid.

Therefore, we have conducted laboratory in situ Raman monitoring experiments in which the $\eta$-value was changed by varying the added liquid volumes. With $9.2 \mu \mathrm{L}$, which is the volume originally used for $1-\mathrm{BuOH}$, and $20 \mu \mathrm{L}$, other lower alcohols and also water gave III as the final product (Table 2). However, an increased preference for I and II was observed when using smaller 
Table 2 Reaction paths and polymorph selectivity in the mechanochemical 1:1 na:ba cocrystal formation.

\begin{tabular}{|c|c|c|c|}
\hline \multirow[b]{2}{*}{ Liquid } & \multicolumn{3}{|c|}{ Volume $(\mu \mathrm{L})$} \\
\hline & 4.1 & 9.2 & 20 \\
\hline $\mathrm{H}_{2} \mathrm{O}$ & $\mathbf{I I}^{a}$ & II $^{a} \longrightarrow$ III & $\mathrm{II}^{a} \longrightarrow \mathrm{III}$ \\
\hline $\mathrm{MeOH}$ & IV $\longrightarrow$ II & II $^{a} \longrightarrow$ III & II $^{a} \longrightarrow$ III \\
\hline EtOH & $\mathbf{I I}^{a} \longrightarrow \mathbf{I}$ & $\mathrm{IV} \longrightarrow \mathrm{II} \longrightarrow \mathrm{III}$ & $\mathrm{IV} \longrightarrow \mathrm{II} \longrightarrow \mathrm{III}$ \\
\hline $\mathrm{PrOH}$ & $\mathbf{I I}^{a} \longrightarrow \mathbf{I}$ & $\mathrm{IV} \longrightarrow \mathrm{II} \longrightarrow \mathrm{III}$ & $\mathrm{IV} \longrightarrow \mathrm{II} \longrightarrow \mathrm{III}$ \\
\hline $\mathrm{BuOH}$ & $\mathrm{II}^{a} \longrightarrow \mathbf{I}$ & $\mathrm{IV} \longrightarrow \mathrm{II} \longrightarrow \mathrm{III}$ & $\mathrm{IV} \longrightarrow \mathrm{II} \longrightarrow \mathrm{III}$ \\
\hline Hexane & III $\longrightarrow$ I & $\mathrm{III} \longrightarrow \mathrm{I}$ & III $\longrightarrow$ I \\
\hline $\mathrm{CH}_{3} \mathrm{CN}$ & I & II $\longrightarrow$ I & $\mathrm{IV} \longrightarrow \mathrm{II} \longrightarrow \mathrm{I}$ \\
\hline
\end{tabular}

${ }^{a}$ Formation of IV before II is likely but could not be ascertained from in situ Raman monitoring.

volumes. For example, using $4.1 \mu \mathrm{L}$ of either $1-\mathrm{BuOH}$, 1-PrOH or EtOH resulted in the formation of $\mathbf{I}$. On the other hand, LAG with $4.1 \mu \mathrm{L}$ of water resulted in the formation of II, analogous to what was seen with the same volume of $\mathrm{MeOH}$.

To better understand the role of the hydroxyl group, we have performed laboratory Raman monitoring experiments of LAG reactions using hexane as a pure aliphatic hydrocarbon liquid. Using up to $20 \mu \mathrm{L}$ of hexane, we observed the same reaction pathway as in NG, yielding initially III and finally I (Table 2, Fig. S21). Phase III was stable in both NG and LAG with hexane while na and ba were present after which the III $\longrightarrow$ I transformation began immediately. This suggested surface nucleation of I which was prevented during growth of phase III.

We have finally performed in situ monitoring using acetonitrile as a polar, but aprotic liquid. Acetonitrile could disrupt hydrogen bonds in ba and na but possibly to a lesser extent then alcohols since it can serve only as a hydrogen-bond acceptor. Indeed, with acetonitrile we have observed a new reaction pathway which depended on the added volume but the final product was always I. With $4.1 \mu \mathrm{L}$ of $\mathrm{CH}_{3} \mathrm{CN}$ we have observed direct formation of I from na and ba (Fig. 8). With $9.2 \mu \mathrm{L}$, intermediate II was formed directly from reactants and transformed later on to I. Finally, using $20 \mu \mathrm{L}$ of $\mathrm{CH}_{3} \mathrm{CN}$, the intermediate II was preceded by phase IV before the final formation of I. Thus, the hydroxyl group is not necessary for the formation of IV and other liquids with hydrogenbonding capabilities can also catalyze its formation. On the other hand, the hydroxyl moiety seems crucial for the stabilization of III.

\section{Conclusion}

In summary, tandem in situ monitoring via PXRD and Raman spectroscopy of the mechanochemical cocrystallisation of na and ba revealed a rich polymorphic system with four new cocrystal phases, multiple phase transformations and a variety of reaction pathways that can be controlled by a careful choice of reaction conditions. The reaction pathway and selectivity can be altered and controlled by the type of the liquid additive in LAG reactions as well as by its amount. The reaction kinetics were quantitatively

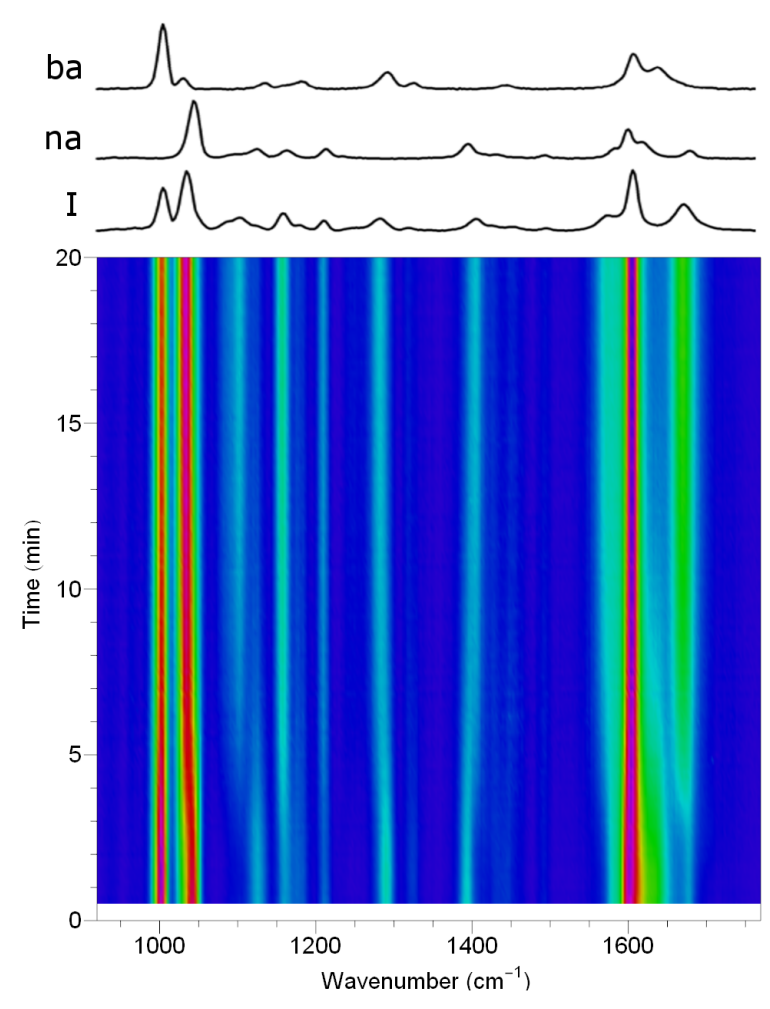

Fig. 8 Raman in situ monitoring of 1:1 na:ba LAG cocrystallization using $4 \mu \mathrm{L}$ of acetonitrile as the liquid additive. The reaction yields directly polymorph $\mathbf{I}$ as the final product.

assessed from tandem in situ monitoring, performed in secondstime resolution, even in situations where not all crystalline phases were structurally characterized. We found reactant depletion to be well described by the first order reaction rate law kinetics while product formation was usually an autocatalytic process.

We also introduce here quantitative in situ Raman monitoring as a technique that is readily implemented in a conventional laboratory as opposed to PXRD monitoring that can be performed only at dedicated synchrotron beamlines. In systems where the reaction mixture is highly crystalline, quantification via PXRD or Raman monitoring will provide similar results but we anticipate that quantitative Raman monitoring could be used to characterize amorphous phases which are known to occur during milling of pharmaceutical materials ${ }^{6,59}$ and in solid-state reactions in general. Our results provide an important first step toward complete quantification of mechanochemical reaction kinetics and emphasize in situ monitoring as necessary analytical tools for understanding of mechanochemical reactivity. The ability to describe reaction kinetics by the simple order-based and Prout-Tompkins reaction models hints the possibility that mechanochemical reactions may bear stronger similarities to solution reactions than could be expected for reactions of solids. By establishing these procedures as a standard in mechanochemical synthesis, we anticipate they will provide the basis for the development of kinetic models of milling reactions and enable formulation of a general mechanistic framework of these increasingly important reactions. 


\section{Acknowledgements}

We are grateful to Mr. Vitomir Stanišić, Mr. Ivan Kulcsár and their team at the Ruđer Bošković Institute for continuous support and Mr. Hrvoje Dagelić for programming. Dr. Dejan-Krešimir Bučar, Dr. Dubravka Šišak and Dr. Manda Ćurić are acknowledged for critically reading the manuscript. Financial support from the ESRF and the Croatian Science Foundation (Grant No. UIP-2014-09-4744) is gratefully acknowledged. IH is grateful to the Adris foundation for supporting this work. SL is supported by the Croatian Science Foundation. TF acknowledges the financial support of the NSERC Discovery Grant and the McGill University W. J. Dawson Scholarship.

\section{References}

1 S. L. James, C. J. Adams, C. Bolm, D. Braga, P. Collier, T. Friščić, F. Grepioni, K. D. M. Harris, G. Hyett, W. Jones, A. Krebs, J. Mack, L. Maini, A. G. Orpen, I. P. Parkin, W. C. Shearouse, J. W. Steed, D. C. Waddell, Chem. Soc. Rev. 2012, 41, 413-447.

2 J. G. Hernandez, C. Bolm, J. Org. Chem. 2017, 82, 4007-4019.

3 D. Braga, L. Maini, F. Grepioni, Chem. Soc. Rev. 2013, 42, 7638-7648.

4 T. Friščić, W. Jones, Cryst. Growth Des. 2009, 9, 1621-1637.

5 C. Aakeröy, Acta Cryst. 2015, B71, 387-391.

6 I. Halasz, A. Puškarić, S. A. J. Kimber, P. J. Beldon, A. M. Belenguer, F. Adams, V. Honkimäki, R. E. Dinnebier, B. Patel, W. Jones, V. Štrukil, T. Friščić, Angew. Chem. Int. Ed. 2013, 52, 11538-11541.

7 M. K. Corpinot, S. A. Stratford, M. Arhangelskis, J. Anka-Lufford, I. Halasz, N. Judaš, W. Jones, D.-K. Bučar, CrystEngComm 2016, 18, 5434-5439.

8 S. Aitipamula, P. Chow, R. Tan, CrystEngComm 2014, 16, 3451-3465.

9 E. Losev, E. Boldyreva, CrystEngComm 2014, 16, 3857-3866.

10 F. Fischer, G. Scholz, S. Benemann, K. Rademann, F. Emmerling, CrystEngComm 2014, 16, 8272-8278.

11 A. M. Belenguer, G. I. Lampronti, A. J. Cruz-Cabeza, C. A. Hunter, J. K. M. Sanders, Chem. Sci. 2016, 7, 6617-6627.

12 A. V. Trask, N. Shan, W. D. S. Motherwell, W. Jones, S. Feng, R. B. H. Tan, K. J. Carpenter, Chem. Commun. 2005, 880-882.

13 D. Hasa, E. Miniussi, W. Jones, Cryst. Growth Des. 2016, 16, 45824588.

14 F. Fischer, A. Heidrich, S. Greiser, S. Benemann, K. Rademann, F. Emmerling, Cryst. Growth Des. 2016, 16, 1701-1707.

15 L. Takacs, Chem. Soc. Rev. 2013, 42, 7649-7659.

16 E. Boldyreva, Chem. Soc. Rev. 2013, 42, 7719-7738.

17 A. D. Jodlowski, A. Yépez, R. Luque, L. Camacho, G. de Miguel, Angew. Chem. Int. Ed. 2016, 55, 14972-14977.

18 P. Baláž, M. Achimovičová, M. Baláž, P. Billik, Z. Cherkezova-Zheleva, J. M. Criado, F. Delogu, E. Dutková, E. Gaffet, F. J. Gotor, R. Kumar, I. Mitov, T. Rojac, M. Senna, A. Streletskii, K. Wieczorek-Ciurowa, Chem. Soc. Rev. 2013, 42, 7571-7637.

19 G.-W. Wang, Chem. Soc. Rev. 2013, 42, 7668-7700.

20 K. Užarević, I. Halasz, I. Đilović, N. Bregović, M. Rubčić, D. MatkovićČalogović, V. Tomišić, Angew. Chem. Int. Ed. 2013, 52, 5504-5508.

21 T. Friščić, I. Halasz, P. A. Beldon, A. M. Belenguer, F. Adams, S. A. J. Kimber, V. Honkimäki, R. E. Dinnebier, Nature Chem. 2013, 5, 66-73.

22 D. Gracin, V. Štrukil, T. Friščić, I. Halasz, K. Užarević, Angew. Chem. Int. Ed. 2014, 53, 6193-6197.

23 L. Batzdorf, F. Fischer, M. Wilke, K.-J. r. Wenzel, F. Emmerling, Angew. Chem. Int. Ed. 2015, 54, 1799-1802.

24 K. Užarević, I. Halasz, T. Friščić, J. Phys. Chem. Lett. 2015, 6, 41294140.

25 I. Halasz, T. Friščić, S. A. J. Kimber, K. Užarević, A. Puškarić, C. Mottillo, P. Julien, V. Štrukil, V. Honkimäki, R. E. Dinnebier, Faraday Discuss. 2014, 170, 203-221.
26 K. Užarević, V. Štrukil, C. Mottillo, P. A. Julien, A. Puškarić, T. Friščić, I. Halasz, Cryst. Growth Des. 2016, 16, 2342-2347.

27 X. Ma, W. Yuan, S. E. J. Bell, S. L. James, Chem. Commun. 2014, 50, $1585-1587$.

28 F. Fischer, K.-J. Wenzel, K. Rademann, F. Emmerling, Phys. Chem. Chem. Phys. 2016, 18, 23320-23325.

29 J. Kieffer, D. Karkoulis, Journal of Physics: Conference Series 2013 , 425, 202012

30 I. Halasz, S. A. J. Kimber, P. J. Beldon, A. M. Belenguer, F. Adams, V. Honkimäki, R. C. Nightingale, R. E. Dinnebier, T. Friščić, Nat. Protoc. 2013, 8, 1718-1729.

31 D.-K. Bučar, G. M. Day, I. Halasz, G. G. Z. Zhang, J. R. G. Sander, D. G. Reid, L. R. MacGillivray, M. J. Duer, W. Jones, Chem. Sci. 2013, 4 4417-4425.

32 R. J. Hill, C. J. Howard, J. Appl.Cryst. 1987, 20, 467-474.

33 M. Caprio, Comput. Phys. Commun. 2005, 171, $107-118$.

34 E. J. Sonneveld, J. W. Visser, J. Appl. Cryst. 1975, 8, 1-7.

35 P. H. C. Eilers, H. F. M. Boelens, Leiden University Medical Center Report 2005.

36 A. de Juan, J. Jaumot, R. Tauler, Anal. Methods 2014, 6, 4964-4976.

37 J. Jaumot, A. de Juan, R. Tauler, Chemometr. Intell. Lab. 2015, 140, 1 -12 .

38 N. Schultheiss, A. Newman, Cryst. Growth Des. 2009, 9, 2950-2967.

39 S. Aitipamula, A. Wong, P. Chow, R. Tan, CrystEngComm 2012, 14 8193-8198.

40 S. G. Fleischman, S. S. Kuduva, J. A. McMahon, B. Moulton, R. D. Bailey Walsh, N. Rodriguez-Hornedo, M. J. Zaworotko, Cryst. Growth Des. 2003, 3, 909-919.

41 D. J. Berry, C. C. Seaton, W. Clegg, R. W. Harrington, S. J. Coles, P. N. Horton, M. B. Hursthouse, R. Storey, W. Jones, T. Friščić, N. Blagden, Cryst. Growth Des. 2008, 8, 1697-1712.

42 S. Karki, T. Friščić, W. Jones, CrystEngComm 2009, 11, 470-481.

43 F. Fischer, D. Lubjuhn, S. Greiser, K. Rademann, F. Emmerling, Cryst. Growth Des. 2016, 16, 5843-5851.

44 R. Dubey, G. R. Desiraju, Chem. Commun. 2014, 50, 1181-1184.

45 C. C. Seaton, A. Parkin, C. C. Wilson, N. Blagden, Cryst. Growth Des. 2009, 9, 47-56.

46 H. C. S. Chan, J. Kendrick, M. A. Neumann, F. J. J. Leusen, CrystEngComm 2013, 15, 3799-3807.

47 N. Chieng, M. Hubert, D. Saville, T. Rades, J. Aaltonen, Cryst. Growth Des. 2009, 9, 2377-2386.

48 N. Shan, F. Toda, W. Jones, Chem. Commun. 2002, 2372-2373.

49 J. C. Burley, M. J. Duer, R. S. Stein, R. M. Vrcelj, Eur. J. Pharm. Sci. 2007, 31, $271-276$

50 A. D. Katsenis, P. A., V. Štrukil, C. Mottillo, P. A. Julien, M. H. Užarević, K. Pham, T. O. Do, S. A. J. Kimber, P. Lazić, O. Magdysyuk, R. E. Dinnebier, I. Halasz, T. Friščić, Nat. Commun. 2015, 6, 6662.

51 H. Kulla, M. Wilke, F. Fischer, M. Rollig, C. Maierhofer, F. Emmerling, Chem. Commun. 2017, 53, 1664-1667.

52 A. Khawam, D. R. Flanagan, J. Phys. Chem. B 2006, 110, 1731517328.

53 M. Avrami, J. Chem. Phys. 1939, 7, 1103-1112.

54 M. Avrami, J. Chem. Phys. 1940, 8, 212-224.

55 E. G. Prout, F. C. Tompkins, Trans. Faraday Soc. 1944, 40, 488-498.

56 M. E. Brown, Thermochim.Acta 1997, 300, 93 - 106.

57 T. Friščić, S. L. Childs, S. A. A. Rizvi, W. Jones, CrystEngComm 2009 , $11,418-426$.

58 A. M. Belenguer, G. I. Lampronti, D. J. Wales, J. K. M. Sanders, J.Am. Chem. Soc. 2014, 136, 16156-16166.

59 A. Jayasankar, A. Somwangthanaroj, Z. J. Shao, N. RodríguezHornedo, Pharmaceut. Res. 2006, 23, 2381-2392. 